\title{
Detection of SFTS virus RNA and antibodies in severe fever with thrombocytopenia syndrome surveillance cases in endemic areas of China
}

Xiaoxia Huang ${ }^{1 \dagger}$, Shujun Ding ${ }^{2 \dagger}$, Xiaolin Jiang ${ }^{2}$, Bo Pang ${ }^{2}$, Quanfu Zhang ${ }^{1}$, Chuan $\mathrm{Li}^{1}$, Aqian $\mathrm{Li}^{1}$, Jiandong Li , Mifang Liang ${ }^{1}$, Shiwen Wang ${ }^{1 *}$ and Dexin $\mathrm{Li}^{1 *}$

\begin{abstract}
Background: Severe fever with thrombocytopenia syndrome (SFTS) is a newly identified severe infectious disease caused by SFTS phlebovirus (SFTSV). SFTS monitoring has been carried out since 2010 in mainland China. We analysed the detection results of SFTSV RNA and antibody in SFTS surveillance cases to provide basic data for SFTS diagnosis.

Methods: This study was conducted in Shandong Province. Sera of SFTS surveillance cases were collected to detect SFTSV RNA and antibody by real-time RT-PCR and enzyme-linked immunosorbent assay, respectively. Detection rates were calculated. SPSS 18.0 (Chicago, IL, USA) was used for statistical analysis to compare the detection rates of SFTSV RNA and antibodies among different sera groups.

Results: A total of 374 SFTS surveillance cases were enrolled. Overall, 93.3\% (349/374) of the sera samples were collected within 2 weeks after onset, and 6.7\% (25/374) were collected between 15 days and 45 days. Of these, 183 (48.9\%) were positive for SFTSV RNA. The SFTSV RNA-positive rate peaked (52.2\%) in samples collected $\leq 7$ days after onset and then showed a decreasing trend. The detection rate of SFTSV-specific IgM antibody was 30.5\% (46/151) and was highest in samples collected between 8 and 14 days $(43.3 \%, 26 / 60)$. The positive rate of SFTSV-specific lgG antibody $(17.9 \%, 27 / 151)$ showed an increasing trend with the specimen collection time. In total, $74.8 \%(113 / 151)$ of sera samples had the same SFTSV RNA and IgM antibody detection results. However, 23.2\% (29/125) of SFTSV RNAnegative cases were IgM antibody-positive, and 8.6\% (9/105) of IgM antibody-negative cases were SFTSV RNApositive.
\end{abstract}

Conclusions: SFTSV RNA detection was preferred for SFTSV infection during disease surveillance. For highly suspected SFTS cases, IgM antibody is suggested to make a comprehensive judgement.

Keywords: SFTS, SFTSV antibodies, Surveillance cases

\footnotetext{
* Correspondence: wangsw@ivdc.chinacdc.cn; lidx@chinacdc.cn

${ }^{\dagger}$ Huang Xiaoxia and Ding Shujun contributed equally to this work.

${ }^{1} \mathrm{NHC}$ Key Laboratory of Medical Viruses and Viral Diseases, National Institute

for Viral Disease Control and Prevention, China CDC, Beijing, China

Full list of author information is available at the end of the article
}

(c) The Author(s). 2019 Open Access This article is distributed under the terms of the Creative Commons Attribution 4.0 International License (http://creativecommons.org/licenses/by/4.0/), which permits unrestricted use, distribution, and reproduction in any medium, provided you give appropriate credit to the original author(s) and the source, provide a link to the Creative Commons license, and indicate if changes were made. The Creative Commons Public Domain Dedication waiver (http://creativecommons.org/publicdomain/zero/1.0/) applies to the data made available in this article, unless otherwise stated. 


\section{Background}

Severe fever with thrombocytopenia syndrome (SFTS), which is mainly characterised by fever, thrombocytopenia, and leukocytopenia, is an infectious disease first identified in China in 2009 [1]. Confirmed cases have also been reported in other Asian countries (Japan and South Korea) $[2,3]$. In China, most of the SFTS cases are farmers aged 40-79 years in seven provinces of central and eastern China $[1,4]$. The average fatality rate is nearly $8 \%$, but it varies in different populations, reaching 30\% [5]. Although SFTS is a tick-borne disease, person-to-person transmission caused by direct contact with blood has also been reported [6-8]. It is still a severe threat to public health.

SFTS phlebovirus (SFTSV) in the Phlebovirus genus of the Phenuiviridae family has been identified as the causative agent. Virus RNA detection by real-time RT-PCR and antibody detection by enzyme-linked immunosorbent assay (ELISA) are commonly used to identify virus infection. The former is often used to confirm SFTSV infection. However, a previous study [9] in Henan Province showed an approximately $50 \%$ positive rate of SFTSV RNA in SFTS surveillance cases, and 14\% of cases with SFTSV-specific IgM antibodies were observed in a group of RNA-negative cases. More information is necessary about the detection of SFTSV RNA and antibodies (especially IgM antibody) in the early stage after disease onset.

Shandong Province is a high epidemic area, with 1074 reported SFTS cases between 2011 and 2014, of which nearly $30 \%$ did not have laboratory evidence [4]. The detection results of SFTSV RNA or antibodies in routine SFTS monitoring were not very clear. To fill this gap, we performed SFTSV RNA and antibody detection and analysis on the acute phase sera of SFTS surveillance cases collected in Shandong Province in 2014. The aim was to understand the detection results of SFTSV RNA and antibodies and to explore appropriate conventional laboratory pathogenic detection strategies to provide a pathogenic and serological basis for better diagnosis of SFTS cases.

\section{Methods}

\section{Sample collection}

A total of 374 sera samples were collected from SFTS surveillance cases distributed in 14 cities of Shandong Province in 2014. Here, SFTS surveillance cases were suspected SFTS cases or clinically diagnosed SFTS cases that required further laboratory detection. General information (e.g., gender, age, occupation, and residence type), epidemiological information (e.g., tick bite history) and clinical manifestation (e.g., body temperature, platelet count, leukocyte count, and lymphadenopathy) from each case were extracted from a well-written questionnaire. Specimens were divided into three groups according to sampling days after onset: $\leq 7$ days (Group A), 8-14 days (Group B), and $\geq 15$ days (Group C); Group AB ( $\leq 14$ days) represents Group A plus Group B.

\section{SFTSV RNA detection}

A total of $140 \mu \mathrm{l}$ of serum was used for RNA extraction with the RNeasy Mini Kit (Qiagen, Germany). Real-time RT-PCR was conducted with the SuperScript III Platinum One-Step Quantitative RT-PCR System Kit (Invitrogen, USA). We followed the kit instructions to conduct the experiments. Reaction parameters were $50^{\circ}$ $\mathrm{C}$ for $30 \mathrm{~min}, 95^{\circ} \mathrm{C}$ for $2 \mathrm{~min}$, and then 40 cycles of $95^{\circ}$ $\mathrm{C}$ for $15 \mathrm{~s}$ and $60^{\circ} \mathrm{C}$ for $30 \mathrm{~s}$. The primers and probes located in the L, M, and S segments of SFTSV were from a previous study [10]. The cut-off cycle threshold (Ct) value was set at 35 cycles.

\section{SFTSV antibody detection}

Sera were tested for SFTSV-specific IgM and IgG antibodies using an ELISA kit (Zhongshan Bio-Tech Co., Ltd.) based on the procedures described previously [11]. In brief, $100 \mu \mathrm{l}$ of diluted serum, $100 \mu \mathrm{l}$ of horseradish peroxidase (HRP)-labelled enzyme conjugate, and the chromogenic substrate were added to each well. Finally, the optical density (OD) value was read at $450 \mathrm{~nm}$ with a microplate reader (Thermo). The cut-off value was set at the average OD value of the negative controls plus 0.10 .

\section{Statistical analysis}

SPSS 18.0 (Chicago, IL, USA) was used for statistical analysis. Pearson chi-square test was conducted to compare the detection rates of SFTSV RNA and antibodies among samples collected on different days. The McNemar test was used to compare SFTSV RNA and IgM antibody detection rates in the same samples, and the kappa value was calculated to compare the detection consistency of the two methods.

\section{Ethical statement}

This study was approved by the ethics committee of the National Institute for Viral Disease Control and Prevention, Chinese Center for Disease Control and Prevention. Written informed consent was obtained from the study participants.

\section{Results}

\section{Characteristics of SFTS surveillance cases}

A total of 374 SFTS surveillance cases were enrolled in this study, mainly distributed in Yantai (156), Weihai (78), Weifang (35), Jinan (28), and Zibo (22) of Shandong Province. The median age of participants was 59 years (range, 7-88 years); 52.8\% (197/373) were male; of the 289 cases with occupation information, the top three occupations were farmers $(81.3 \%)$, workers $(5.9 \%)$, and 
household workers and unemployed (3.8\%). Ninety-nine percent had a fever. A total of 93.3\% (349/374) of the samples were collected within 2 weeks after onset, and the remaining $6.7 \%(25 / 374)$ were collected between 15 days and 45 days. The median and interquartile range $(25 \%, 75 \%)$ of the collection days after onset of the 374 samples was 7 days (4-9 days). The major clinical and epidemiological information of the participants is shown in Table 1.

\section{SFTSV RNA detection}

Of the 374 samples, 183 (48.9\%) were positive for SFTSV RNA (Table 2). SFTSV RNA was detected on the day of onset and until the 27th day after onset. The positive rate of SFTSV RNA among Group A was 52.2\%, which decreased with the increase in the time interval between specimen collections. The positive rate of SFTSV RNA in Group C was $28 \%$. There was no statistically significant difference $(\chi 2=0.818, P=0.366)$ between Group A and Group B. However, a significant difference was found in specimens collected between Group AB and Group C ( $\mathrm{x} 2=4.697, P=0.03)$.

\section{SFTSV antibody detection}

Of the 374 cases, 151 sera samples were chosen to conduct SFTSV antibody (IgM and IgG) detection. The median collection time of these specimens was 7 days (range 0-45 days). The detection rate of SFTSV-specific IgM antibody was $30.5 \%$ (46/151). IgM antibodies were detected on the onset day and could still be detected 45 days after onset. The highest IgM antibody detection rate was found in Group B $(43.3 \%, 26 / 60)$. There was a difference in the detection rate of IgM between Group A and Group B ( $X 2=8.865, P=0.003)$. The positive rate of SFTSV-specific IgG antibody was $17.9 \%(27 / 151)$. The detection rate of IgG antibody showed an increasing trend with the sampling days. There was no statistically significant difference in the detection rate of IgG antibodies among Groups $\mathrm{A}, \mathrm{B}$, and $\mathrm{C}\left(\mathrm{x}^{2}=3.048, \mathrm{P}=\right.$ 0.218 ). Table 2 show the antibody detection results.

\section{Comparison of SFTSV RNA and IgM antibody detection}

The detection rates of SFTSV IgM antibodies were $65.4 \%(17 / 26)$ and $23.2 \%(29 / 125)$ in SFTSV RNA-positive and SFTSV RNA-negative samples, respectively (Table 3$)$. A total of $74.8 \%(113 / 151)$ of the samples had the same SFTSV RNA and IgM antibody detection results. However, there was a significant difference between the detection rates of SFTSV RNA and IgM antibody, and the detection consistency was poor.

\section{Discussion}

Pathogen detection mainly targets RNA and antibodies. Real-time RT-PCR is a commonly used method for virus RNA detection, which directly targets the RNA of pathogens. ELISA is often used for antibody detection, which is an indirect method to determine whether virus infection exists by detecting virus antibodies (IgM and $\operatorname{IgG}$ ). The former has a higher detection sensitivity for specimens collected in the acute phase [10]; however, it has higher requirements for the testing environment and skilled personnel. ELISA requires a simpler operating environment and is much easier to operate by professionals at the county level. These two methods are often used in the early diagnosis of pathogens. Therefore, we used these two methods to detect SFTSV RNA and antibodies (IgM and IgG) in this study.

Shandong Province is a highly endemic area for SFTS and where SFTSV RNA detection is mainly used to confirm SFTSV infection during surveillance. Here, we compared the detection rates of SFTSV RNA and SFTSV-specific antibodies. Approximately $49 \%$ of SFTS surveillance cases had SFTSV RNA, and 30.5\% had SFTSV-specific IgM antibodies, which was similar to other recorded data [9]. SFTSV RNA was detected on the onset day, and the detection rate, which was highest within the first week (52.2\%), decreased with sampling time. The detection rate of SFTSV IgM antibody was higher in the second week. The SFTSV RNA detection rate was much higher than that of IgM antibody for samples collected within two weeks after onset. Therefore, SFTSV RNA should be given priority in the early detection of SFTSV. For SFTSV RNA detection, serum

Table 1 Major clinical and epidemiological information of participants

\begin{tabular}{lllll}
\hline Variable & Normal level & Observed no. & No. (\%) & Median and interquartile range $(25 \%, 75 \%)$ \\
\hline Fever $\left(>37.3^{\circ} \mathrm{C}\right)$ & - & 302 & $299(99)$ & NA \\
With gastrointestinal symptoms & - & 288 & $253(87.8)$ & $\mathrm{NA}$ \\
Lymphadenopathy & - & 298 & $56(18.8)$ & $\mathrm{NA}$ \\
With tick bite history & - & 299 & $36(12)$ & $\mathrm{NA}$ \\
With fatigue & - & 302 & $254(84.1)$ & $\mathrm{NA}$ \\
Leukocyte count, $10^{9} / \mathrm{L}$ & $4-10$ & 257 & $\mathrm{NA}$ & $2.7(1.9-4.6)$ \\
Platelet count, $10^{9} / \mathrm{L}$ & $100-300$ & 254 & $\mathrm{NA}$ & $69(47-92)$ \\
\hline
\end{tabular}


Table 2 SFTSV RNA, IgM and IgG antibody detection results

\begin{tabular}{llll}
\hline Sampling days & $\begin{array}{l}\text { SFTSV RNA-positive samples no. / total } \\
\text { detection no. (\%) }\end{array}$ & $\begin{array}{l}\text { SFTSV IgM antibody-positive } \\
\text { samples no. / total detection no. (\%) }\end{array}$ & $\begin{array}{l}\text { SFTSV IgG antibody-positive } \\
\text { samples no. / total detection no. (\%) }\end{array}$ \\
\hline $\mathbf{3}$ days & $119 / 228(52.2)$ & $15 / 76(19.7)$ & $11 / 76(14.5)$ \\
$8-14$ days & $57 / 121(47.1)$ & $26 / 60(43.3)$ & $11 / 60(18.3)$ \\
$\geq 15$ days & $7 / 25(28)$ & $5 / 15(33.3)$ & $5 / 15(33.3)$ \\
Total & $183 / 374(48.9)$ & $46 / 151(30.5)$ & $27 / 151(17.9)$ \\
\hline
\end{tabular}

collection during the acute phase (within two weeks after disease onset) of disease was often recommended; therefore, we compared the SFTSV RNA detection and IgM antibody detection results in two groups $(\leq 14$ days and $\geq 15$ days). This study showed that the detection rates between SFTSV RNA and IgM were different, and the detection consistency was poor. Nearly a quarter of the cases negative for SFTSV RNA were positive for SFTSV IgM antibody, which was not observed in healthy people [12]. These results implied that for SFTSV RNA-negative cases, exclusion should be carefully considered. In addition, $8.6 \%$ of SFTSV IgM antibody-negative cases were positive for SFTSV RNA. Therefore, SFTSV IgM antibody-negative cases could not be completely ruled out as SFTS when only relying on IgM antibody detection. The positive rate of SFTSV IgG antibody was only $17.9 \%$, and it maintained at a relatively low level within one and a half months after disease onset. Paired specimens were required for SFTSV infection confirmed with IgG antibody, which was difficult to collect; therefore, IgG antibody testing was generally not used for early routine case monitoring.

SFTSV RNA detection was superior to IgM antibody detection; however, undetected phenomenon in real SFTS cases may exist both in SFTSV detection alone and in SFTSV IgM detection alone. It has been reported that the IgM antibody-positive rate was very high in confirmed SFTS cases $[9,13]$. However, because IgM antibodies exist for a long time after onset [14], SFTSV IgM antibody positivity alone might not be sufficient evidence for current infection. Therefore, it is suggested that SFTSV RNA detection is preferred for SFTSV

Table 3 Comparison of SFTSV RNA and IgM antibody detection

\begin{tabular}{llllll}
\hline $\begin{array}{lllll}\text { Days after } \\
\text { onset }\end{array}$ & $\begin{array}{l}\text { SFTSV } \\
\text { RNA }\end{array}$ & \multicolumn{2}{l}{ SFTSV IgM } & $\begin{array}{l}\text { P value } \\
\text { (McNemar test) }\end{array}$ & $\begin{array}{l}\text { Kappa } \\
\text { value }\end{array}$ \\
\cline { 3 - 5 } & & Positive & Negative & & \\
\hline S14 days & Positive & $15(62.5)$ & $9(37.5)$ & 0.06 & 0.307 \\
& Negative & $26(23.2)$ & $86(76.8)$ & & \\
\multirow{2}{*}{$\geq 15$ days } & Positive & $2(100)$ & $0(0)$ & 0.25 & 0.471 \\
& Negative & $3(23.1)$ & $10(76.9)$ & & \\
\multirow{2}{*}{ Total } & Positive & $17(65.4)$ & $9(34.6)$ & 0.002 & 0.323 \\
& Negative & $29(23.2)$ & $96(76.8)$ & & \\
\hline
\end{tabular}

infection during disease surveillance. For highly suspected SFTS cases, testing for IgM antibody is suggested to make a comprehensive judgement.

To interpret the results of the study, the following limitations should be considered: first, for the detection rate analysis, three groups (0-7 days, $8-14$ days and $\geq 15$ days) were divided according to sampling days. The $\geq 15$ days group had a relatively small number of cases, and the time interval between specimen collection and onset was relatively wide (15-45 days), which might cause bias in the statistical analysis. Second, considering that there might be missed SFTS cases in the SFTSV RNA-negative cases, more SFTSV RNA-negative cases were chosen to conduct antibody detection, which might lead to a certain bias in the detection rate comparison between these two methods.

\section{Conclusions}

By analysing the detection rate of SFTSV RNA and antibodies conducted with real-time RT-PCR and ELISA, our study suggested that SFTSV RNA testing was the preferred method during SFTS surveillance. However, for highly suspected cases that are SFTSV RNA-negative, case exclusion should be carefully considered, and IgM antibody detection is suggested to provide further information for case exclusion.

\section{Abbreviations}

ELISA: Enzyme-linked immunosorbent assay; SFTS: Severe fever with thrombocytopenia syndrome; SFTSV: SFTS phlebovirus

\section{Acknowledgements}

The authors thank the staff at the hospitals and local centres for disease control and prevention in Shandong Province for their data and specimen collection.

\section{Funding}

This work was supported by the China Mega-Project for Infectious Diseases (2018ZX10711001, 2017ZX10303404-007-002), the National Natural Science Foundation of China (Grant No. 81102171), the National Key Research and Development Program of China (2016YFC12002), and the Natural Science Foundation of Shandong Province (Grant No. ZR2014HP030). The funders had no role in study design, data collection and analysis, data interpretation, and manuscript writing.

\section{Availability of data and materials}

The datasets generated and/or analysed during the current study are not publicly available due to consent for data sharing not being obtained at enrolment, but aggregate data are available from the corresponding author on reasonable request. 


\section{Authors' contributions}

Study design, reagent support: DXL, SWW, MFL, JDL, XXH, AQL. Data and specimen collection: SJD, XLJ, BP. Experiments and data analysis: XXH, SJD, $X L J, B P, Q F Z$, CL. Writing: XXH. All authors read and approved the final manuscript.

\section{Ethics approval and consent to participate}

This study was approved by the ethics committee of the National Institute for Viral Disease Control and Prevention, Chinese Center for Disease Control and Prevention. Written informed consent was obtained from the study participants.

\section{Consent for publication}

Not applicable.

\section{Competing interests}

The authors declare that they have no competing interests.

\section{Publisher's Note}

Springer Nature remains neutral with regard to jurisdictional claims in published maps and institutional affiliations.

\section{Author details}

${ }^{1} \mathrm{NHC}$ Key Laboratory of Medical Viruses and Viral Diseases, National Institute for Viral Disease Control and Prevention, China CDC, Beijing, China.

${ }^{2}$ Shandong Provincial Key Laboratory of Communicable Disease Control and Prevention, Shandong Center for Disease Control and Prevention, Shandong Province, Jinan, China.

Received: 14 October 2018 Accepted: 7 May 2019

Published online: 28 May 2019

\section{References}

1. Li D. A highly pathogenic new bunyavirus emerged in China. Emerg Microbes Infect. 2013;2(1):e1.

2. Takahashi T, Maeda K, Suzuki T, Ishido A, Shigeoka T, Tominaga T, Kamei T, Honda M, Ninomiya D, Sakai T, et al. The first identification and retrospective study of severe fever with thrombocytopenia syndrome in Japan. J Infect Dis. 2014;209(6):816-27.

3. Chang MS, Woo JH. Severe fever with thrombocytopenia syndrome: tickmediated viral disease. J Korean Med Sci. 2013;28(6):795-6.

4. Li Y, Zhou H, Mu D, Yin W, Yu H. Epidemiological analysis on severe fever with thrombocytopenia syndrome under the national surveillance data from 2011 to 2014, China. Zhonghua Liu Xing Bing Xue Za Zhi. 2015;36(6): 598-602.

5. Yu XJ, Liang MF, Zhang SY, Liu Y, Li JD, Sun YL, Zhang L, Zhang QF, Popov $\mathrm{VL}, \mathrm{Li}$ C, et al. Fever with thrombocytopenia associated with a novel bunyavirus in China. N Engl J Med. 2011;364(16):1523-32.

6. Chen H, Hu K, Zou J, Xiao J. A cluster of cases of human-to-human transmission caused by severe fever with thrombocytopenia syndrome bunyavirus. Int J Infect Dis. 2013;17(3):e206-8.

7. Tang X, Wu W, Wang H, Du Y, Liu L, Kang K, Huang X, Ma H, Mu F, Zhang S, et al. Human-to-human transmission of severe fever with thrombocytopenia syndrome bunyavirus through contact with infectious blood. J Infect Dis. 2013;207(5):736-9.

8. Jiang XL, Zhang S, Jiang M, Bi ZQ, Liang MF, Ding SJ, Wang SW, Liu JY, Zhou SQ, Zhang XM, et al. A cluster of person-to-person transmission cases caused by SFTS virus in Penglai, China. Clin Microbiol Infect. 2015;21(3):274-9.

9. DU YH, XY HUANG, HF WANG, AG YOU, KANG K, HM CHEN, BL XU. Comparison on real-time PCR and ELISA methods in detection of severe fever with thrombocytopenia syndrome (SFTS) cases. CHINESE JOURNAL OF ZOONOSES. 2013;29(1):101-4

10. Sun Y, Liang M, Qu J, Jin C, Zhang Q, Li J, Jiang X, Wang Q, Lu J, Gu W, et al. Early diagnosis of novel SFTS bunyavirus infection by quantitative realtime RT-PCR assay. Journal of clinical virology: the official publication of the Pan American Society for Clinical Virology. 2012;53(1):48-53.

11. Huang X, Zhang Z, Jin G, Wang X, Tan C, Yin H, Wang S. Presence of antibodies against severe fever with thrombocytopenia syndrome virus in non-endemic areas, China. Jpn J Infect Dis. 2017;70:248-51.
12. xiaoli D, jianbo Z. Survey on the infectious status of population with severe fever with thrombocytopenia syndrome bunyavirus in Macheng City, Hubei Province in 2013. Practical Preventive Medicine. 2014;21(06):681-2.

13. Liu Y, Sun TT, Zhang J, Wang ZJ, Yao WQ, Zhao Z. Three methods for detecting severe fever with thrombocytopenia syndrome bunyavirus. Chinese Journal of Zoonoses. 2014;30(11):1129-32.

14. Li dx. Fever with thormbocytopenia associated with a novel Bunyavirus in China. Chin J Exp Clin Virol. 2011;25(2):81-4

\section{Ready to submit your research? Choose BMC and benefit from:}

- fast, convenient online submission

- thorough peer review by experienced researchers in your field

- rapid publication on acceptance

- support for research data, including large and complex data types

- gold Open Access which fosters wider collaboration and increased citations

- maximum visibility for your research: over $100 \mathrm{M}$ website views per year

At BMC, research is always in progress.

Learn more biomedcentral.com/submissions 